\title{
Suicide Rate in Oman in the Period from January 2000 to December 2010
}

\author{
Saif Al Hashmi, Ahmed Al Sabri and Nasser Al Felati \\ Armed Forces Hospital, Seeb, Sultanate of Oman
}

\begin{abstract}
Background: suicide rate has been explored in different part of the world, including many Arab /Islamic countries. To our knowledge, no study has been forthcoming from Oman. Aim: To examine the rate of suicide in Sultanate of Oman in a ten year period (2000-2010). Method: Data kept at the Royal Oman Police Forensic Department was scrutinized for the presence of suicide causes of death for 10 year period (2000-2010). Results: Total number suicide cases in Oman in that period were 599 cases, with 59.9 cases every year. Omanis account for 8.7 cases every year. Conclusion: Suicide rate in Oman was found to be 2/100,000/year. This makes Oman to be in the group of low suicide rates in the world.
\end{abstract}

Key words: Suicide, Oman, self harm.

\section{Background}

Suicide is an act of taking one's own life. Suicidal behavior is any action that could cause a person to die, such as taking a drug overdose or crashing a car on purpose [3]. According to calculations based on data reported to WHO by its Member States, in 1998 suicide represented $1.8 \%$ of the global burden of disease and it is expected to increase to $2.4 \%$ by the year 2020. Suicide is among the 10 leading causes of death for all ages in most of the countries for which information is available. In some countries, it is among the top three causes of death for people aged 15-34 years. In the year 2020, according to WHO's projections; approximately 1.53 million people will die from suicide 10 to 20 times more people will attempt suicide.

This represents on average one death every 20 seconds and one attempt every 1-2 seconds [2].

The Omani culture owes much to the geography of the country. The cultural heartland lies in the interior, in the valleys of the mountainous backbone which

Corresponding author: Saif Salam AL Hashmi, MD, research fields: PTSD, sleep and suicide. E-mail: nouralmanar@gmail.com. parallels the coastal plains and the interior plains. Seas to the north and east and deserts to west and south have served to isolate the country from the outside world before 1970. At the same time, Oman's presence on the Indian Ocean has fostered a long maritime tradition which has enriched the culture through the settlement of many Indo-Iranian people of Baluchistan and along the northern coast the interaction with East African cultures. Traditionally, Oman's capital was located in the interior but Muscat, now the principal seaport, has served as the capital since the beginning of the nineteenth century. Northern Oman is separated from southern Dhofar by several hundred miles of desert, which results in the cultural distinctiveness of the people from the south [8].

\section{Aim}

To examine the rate of suicide in Sultanate of Oman in a ten year period (2000-2010).

\section{Method}

Data collected from the Royal Oman Police Forensic Department .We looked at the total number of suicide cases that were reported officially to the 
department in a ten years interval from the year 2000 till 2010.

The targeted population used in the study is the general population that is officially published in the census done in 2010, which were roughly 3,000,000 [7]. That is because studies in literature reporting suicide rate to WHO used general population.

Related important variables were also looked at:

(1) Sexual variation

(2) Regional distribution (Oman is divided into 9 regions. Muscat region is the capital and the second most populated region after al Batinah region, with a total population around 734,697 (census 2010). The cases were distributed according to the frequency of occurrence in each region.

(3) Year and sex distribution

(4) Age groups

(5) Victims nationality was divided into four groups of countries;

East Asia: including (Bangladesh, Indians, Indonesians, Nepalan, Pakistani, Sirilanka, Philippine,)

Europeans: (British, German, Irish, Swiss, Turkish)

Africans: (Ethiopian, Moroccan)

Unknown

Omanis were put in one separate category

(6) Method of suicide

(7) Place of occurrence was divided into three main categories:

Indoor: (suicide cases took place indoor such as; room, kitchen, toilet, ect.)

Outdoor: (suicide cases took place outdoor, such as; wadi, on a tree, farm, etc.)

Unknown: (no information of the place of occurrence

(8) Body part affected (denotes to the most important body parts affected by the act of suicide).

(9) Occupation of victim which was categorized into four main categories;

Professional: (doctor, professor, accountant, nurse,)

Student:
Others: (non professional such as; laborer, driver, welder),

Unknown: (no information available)

\section{Results}

The total number of cases was 599 over the ten years period. In term of gender approximately $16 \%$ (n = 97) were female and the rest were males (502) (Table 1, Fig. 1).

\section{Table 1 Gender of suicide.}

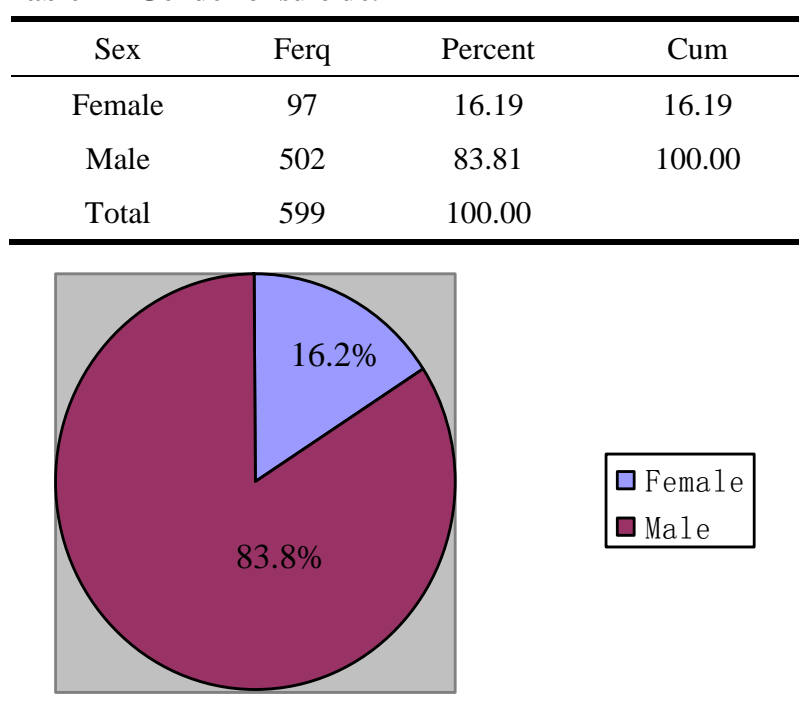

Fig. 1 Gender of the victims (distribution of cases according to the year, sex).

Table 2 Years and gender of suicide.

\begin{tabular}{cccc}
\hline Year & Female & Male & Total \\
\hline 2000 & 7 & 31 & 38 \\
2001 & 8 & 49 & 57 \\
2002 & 9 & 38 & 47 \\
2003 & 9 & 44 & 53 \\
2004 & 9 & 40 & 49 \\
2005 & 7 & 50 & 57 \\
2006 & 6 & 44 & 50 \\
2007 & 9 & 49 & 58 \\
2008 & 15 & 51 & 66 \\
2009 & 8 & 57 & 65 \\
2010 & 10 & 49 & 59 \\
Total & 97 & 502 & 599 \\
\hline
\end{tabular}




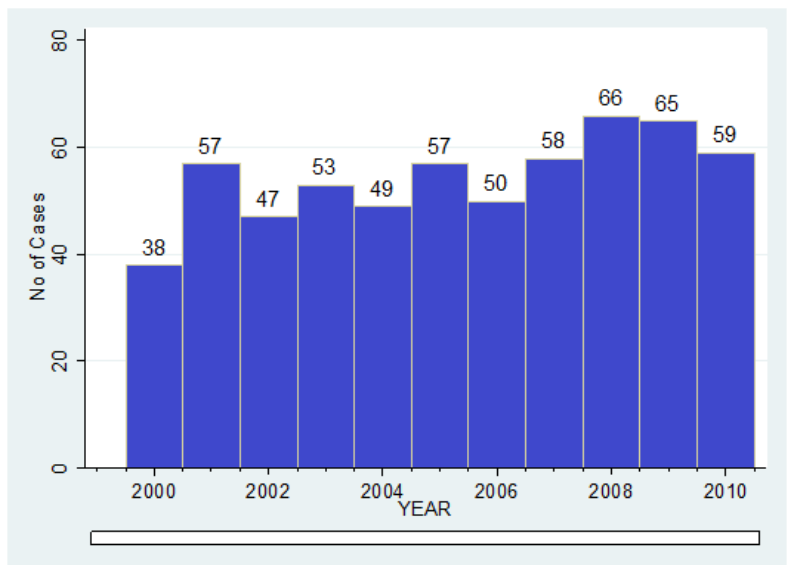

Fig. 2 Histogram of cases by year (2000-2010): age groups (in years).

Table 3 Ages of suicide.

\begin{tabular}{cccc}
\hline Grouped age & Freq & Percent & Cum \\
\hline$[0-10]$ & 65 & 10.85 & 10.85 \\
{$[11-20]$} & 215 & 35.89 & 46.74 \\
{$[21-30]$} & 183 & 30.55 & 77.3 \\
{$[31-40]$} & 94 & 15.69 & 92.99 \\
{$[41-50]$} & 25 & 4.17 & 97.16 \\
{$[51-60]$} & 17 & 2.84 & 100.00 \\
Total & 599 & 100.00 & \\
\hline
\end{tabular}

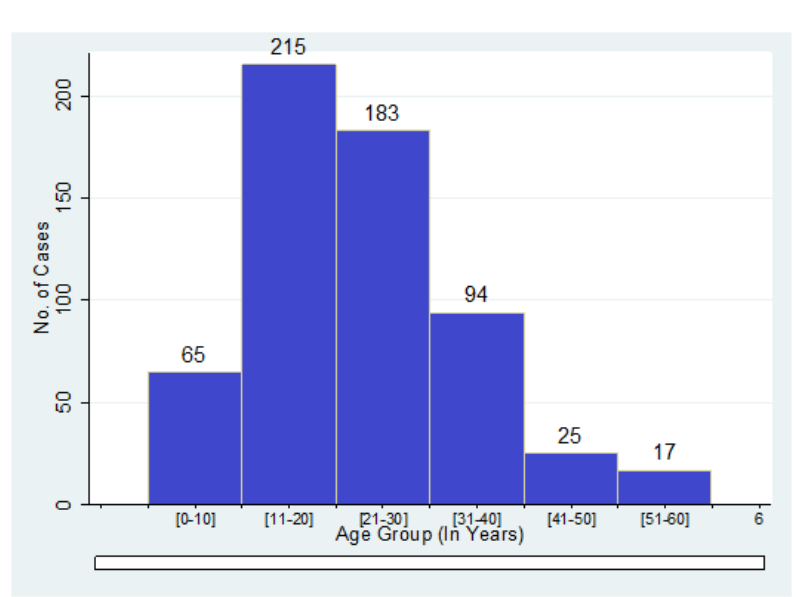

Fig. 3 Victims according to the nationality: East Asia: 491; Omani: 87; Europeans: 5; Africans: 4; Middle East: 2; Unknown: 10.

\subsection{Methods of Suicide (Table 3)}

\subsection{Place of Occurrence}

Indoor: 456 (Suicide cases took place indoor such as; room, kitchen, toilet, ect.)
Outdoor: 82 (suicide cases took place outdoor, such as; wadi, on a tree, farm... etc.)

Unknown: 60

-(no information of the place of suicide were available.

\subsection{Body Part Mostly Affected}

Body parts most affected (Fig. 4):

-Hanging/ cut wounds: Neck 537, hand 1

-Stab wounds/ gunshot wounds: Abdomen 5, Chest 14, Head 10, Mouth 10, Under chin 1

-Others (burns, drowning, poisoning): 21

\subsection{Occupation}

Occupation:

Professional: 8 (doctor, professor, accountant, nurse,)

Student: 3

Table 4 Type of suicide.

\begin{tabular}{cccc}
\hline Type & Freq & Percent & Cum \\
\hline Burn & 4 & 0.67 & 0.42 \\
Cut neck & 1 & 0.17 & 0.83 \\
Cut Throat & 1 & 0.17 & 1 \\
Cut Wrist & 1 & 0.17 & 1.17 \\
Drowning & 2 & 0.33 & 1.5 \\
Fall & 6 & 1 & 2.5 \\
Gunshot & 32 & 5.34 & 7.85 \\
Hanging & 531 & 88.65 & 96.49 \\
Poisoning & 19 & 3.17 & 99.67 \\
Strangulation & 2 & 0.33 & 100 \\
Total & 599 & 100 & \\
\hline
\end{tabular}

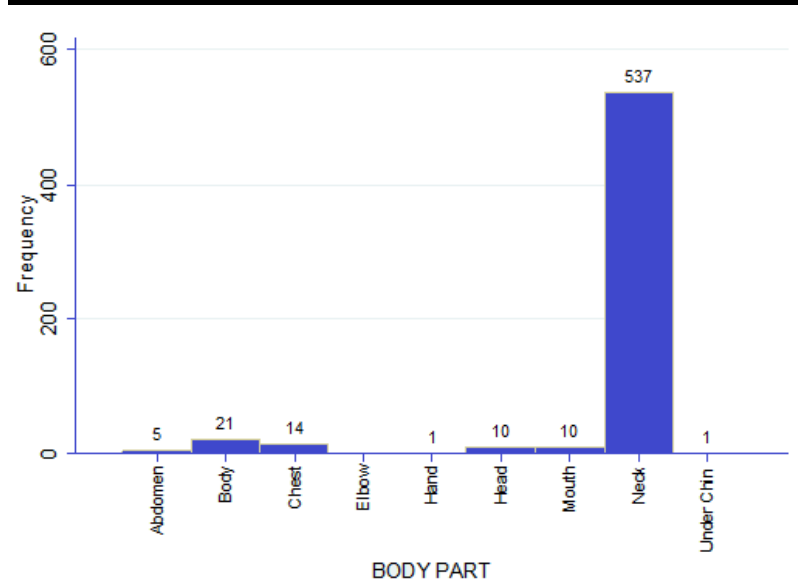

Fig. 4 Histogram of body parts mostly affected. 
Others: 494 (non professional such as; laborer, driver, welder...)

Unknown: 111 (no information available)

\subsection{Regions in Oman}

Table 5 Regions of suicide.

\begin{tabular}{cccc}
\hline Region & Freq & Percent & Cum \\
\hline BATINAH & 125 & 20.87 & 20.87 \\
BURAIMI & 12 & 2.00 & 22.87 \\
DAKHELIYA & 56 & 9.35 & 32.22 \\
DHAHIRA & 43 & 7.18 & 39.40 \\
DHOFAR & 13 & 2.17 & 41.57 \\
MUSANDAM & 8 & 1.34 & 42.90 \\
MUSCAT & 257 & 42.90 & 85.81 \\
SHARKIYA & 77 & 12.85 & 98.66 \\
WOUSTA & 8 & 1.34 & 100.00 \\
Total & 599 & 100 & \\
\hline
\end{tabular}

\section{Discussions}

To our knowledge this is the first study showing national trend of suicide in Oman.

A search was conducted on several search engines (Pub Med, Psychinfo, IDRAAC WEB/CD) up to 2006 (Bahrain, Egypt, Iraq, Jordan, Kuwait, Lebanon, Morocco, Palestine, Saudi Arabia, Sudan and United Arab Emirates). Results varied across countries and methods. In Arab Community studies, the prevalence of lifetime suicide ideation varied from a low of $2.09 \%$ to a high of $13.9 \%$ and the lifetime prevalence of attempts from $0.72 \%$ to $6.3 \%$ [9].

The total number of cases was 599, of which 97 were females and 502 were males, with ratio of 1 female to 5 males.

The average number of suicide cases is 59.9 case/year/population (around 3,000,000 in the latest consensus in 2011).

This makes our suicide rate per 100,000 per year to be 1.9966 .

The lowest number of suicide cases was in the year 2000, with 38 cases and the highest was in 2008 with 66 cases.
The total number of suicide cases in females remained between 7-10/year, with only one peak in the year 2008, which went up to 15 cases.

Whereas in males it was in the range between, the lowest of 31 cases in the year 2000, to the highest in the year 2009 with 57 cases.

Suicidal behavior included suicide ideation, suicide attempts, and suicide completions, as well as suicide-prone behaviors. Across most countries, females have higher rates of suicide ideation and more frequent suicide attempts than males; females also score higher than males on measures of suicidality that overlap with depression assessments. However, males generally have higher rates of suicide completions.

In the United States, across most age groups, men complete suicide more often than women, yet women attempt suicide more often than men.

The trend for males to complete suicide more than females was also found in all but one of the 56 countries catalogued by Lester (1997). Furthermore, Lester (1997) concluded, "While male suicide rates seem to be rising worldwide, females rates do not.”

These findings suggest that sex differences in rates of suicide completion's are becoming more pronounced over time (males greater than females) [10].

The present study also examined regional differences in suicide patterns. Most of suicides were in urban regions. Muscat region and the surrounding northern region of Oman is the most populated regions of Oman. Being urbanized part of the country, these are the areas where most of the foreign labor are living. This explains why these regions have high number of suicide.

For many decades the states of the Gulf Cooperation Council have served as primary migratory destinations for tens of millions of individuals from South Asia, West Asia, and other points in the Indian Ocean world. For tens of millions of families in South Asia, the Middle East, and portions of East and North Africa, the petroleum-rich 
states of the Arabian Peninsula present the most significant migratory waypoint in the spectrum of possibilities they face [11].

The most affected group was adolescents between the ages of 11-20 years with total of 215 cases accounting for $35.89 \%$ of total cases in the ten years period. This was followed by young adults, between the ages of 21-30 years old, with 183 cases forming $30.55 \%$ of the total meaning that age group between $11-30$ accounts for $66.4 \%$ of the whole.

Looking at the nationality of victims, majority were Indian nationals with 415 cases forming $68.28 \%$ of the whole.

Omanis accounted for $14.25 \%$ with 87 cases over the ten years period, with annular average rate of 8.7 cases/year/population.

The methods used in suicide varied, majority used hanging in 531 cases with $88.65 \%$ of the total. This explains why the neck was the most affected of the body, and why the rope was the most frequent mean used in 293 cases (48.91\%), followed by use of clothing in 174 cases (29.85\%). Majority of suicides occurred in a closed room, 322 cases (53.85\%) Suicide was most frequent in people working as laborers with 383 cases (64.05\%), which are expected as Oman is a developing petroleum nation of small population, and the importing of cheap, low class workers is the mandatory for development. These laborers are mostly from India, and Sirilanka. As well as majority of housemaids are from Indonesia.

The regional distribution of suicide showed that it was more prevalent in the capital area Muscat with 257 cases (42\%), followed by the Batinah region with 125 cases (20.87\%).

In our study there are several points of weakness .Firstly, there are presumably large number of cases which are not reported or no autopsy done to document the nature of death. The other point is that the population used is the general population, which does not really represent the population at risk for suicide since it is not known to occur in the childhood or infancy; nevertheless they represent large percentage of the general population. Unfortunately all studies that are found in the literature, reporting suicide rate do that. The other thing is that the population used is the one published in the census done at the end of 2010, but the study is looking at the rate over ten years period. Thus the population was not static over that period, and it was lower that what it is now.

Suicide data are reported to the WHO is in absolute numbers along with the mid-year population of a country. The suicide rates are usually represented by country, year, sex, and age group. The official figures made available to WHO by its Member States are based on death certificates signed by legally authorized personnel, usually doctors and, to a lesser extent, police officers [3].

This makes our source of data very reliable, since it was obtained from the forensic department in the police, which is the only authorized agency in the country to issue any death certificate for death resulting from suicide or any other un-natural causes. Thus, suicide may be hidden and underreported for several reasons, e.g., as a result of prevailing social or religious attitudes. Which is well known in some places of the world, it is believed that suicide is underreported by a percentage between $20 \%$ and $100 \%$ ?

Despite this, suicide has received relatively less attention on this part of the world compared to Europe and North America. Lack of resources and competing priorities in many countries has contributed to this under-emphasis. Cultural influences, religious sanctions, stigmatization of the mentally ill, political imperatives, and socio-economic factors have also played a significant role. As a result, the magnitude of the problem will remain underestimated.

According to WHO reports, there are countries with high suicide rate exceeding 27/100,000/year, including Russia, Lithuania, Hungary, and Japan. There are countries with medium high rates of suicide; between 
17-23, like Belgium, Finland, France, S. Korea, .... There are countries of medium suicide rates, ranging from 9-17; including Denmark, Germany, India, United States. The last group is of low suicide rates; less than 9/100000/year, including Spain, Italy, U.K, and Kuwait [6]. Oman would fit in the low suicide rate countries.

Many Arab countries, report having zero suicide rate, which I found indeed very odd. There are always mentally ill people in every population, and a high proportion of depressed people, of which some who will commit suicide.

As found in our study Oman has low suicide rate, which is expected from an Islamic country. Suicide rate is low in Islamic countries, explained by concept of life and views on suicide within Islamic teachings. Religious commitments in Islam, and adherence to the normative structure of collectivism, including collective goals, non egoistic behavior, familial society, and cohesive committees. All may play major role in reducing suicide rates in these countries [5].

\section{Conclusion}

Oman is showing low suicide rate of around 2/100,000/year. Omanis are showing even lower incidence of 8.7 cases/year.

\section{Recommendations}

(1) At the national, it will be essential to repeat the study every $3-5$ years to update the statistics.
(2) Insure proper reporting, and documentation of every case of death.

(3) Need to carry on doing a retrospective analysis, to provide a psychological autopsy of a sample of the documented cases of suicide.

(4) WHO has to ensure that countries have to update their statistics, since most of them are out of date especially in amongst the Arab countries?

\section{References}

[1] Royal Oman Police forensic department data on reported suicide cases from January 2000 to end of 2010.

[2] Suicide and psychiatric diagnosis: A worldwide perspective, Jose Manoel Bertolote, Alexandra FLleischmann, World Psychiatry 1-3 Oct. 2002.

[3] Suicide and Suicide Prevention in Asia, WHO 2008.

[4] J. M. Bertolote, A. Fleischmann, D. De Leo and D. Wasserman, Psychiatric diagnoses and suicide: Revisiting the evidence, 2004.

[5] Ajit Shah and Mahmood Chandia, The relationship between suicide and Islam, Journal of Injury and Violence Research 2 (2) (2010).

[6] E. G. Karam, R. Hajjar and M. Salamoun, Suicidality in the Arab world Part I: Community Studies, Arab Journal of Psychiatry 18 (2) (2007) 99-107.

[7] A Gendered Analysis of Sex Differences in Suicide-Related Behaviors: A National (U.S.) and International Perspective Jennifer LanghinrichsenRohling, Ph.D. University of South Alabama, 2001.

[8] Andrew M. Gardner, Gulf migration and the family, Journal of Arabian Studies 1 (1) (2011) 3-25. 